\title{
Securing Visible Light Communications with Spatial Jamming
}

\author{
Sunghwan Cho*, Gaojie Chen" ${ }^{\#}$, and Justin P. Coon* \\ *Department of Engineering Science, University of Oxford, OX1 3PJ, United Kingdom. \\ \{sunghwan.cho and justin.coon\}@eng.ox.ac.uk \\ \#Department of Engineering, University of Leicester, LE1 7RH, United Kingdom. \\ gaojie.chen@leicester.ac.uk
}

\begin{abstract}
In this paper, we propose a secure visible light communication (VLC) system with a novel spatial jamming scheme, which is inspired by practical observations of indoor VLC environments. In reality, probable and approximate locations of VLC users can be anticipated by analyzing the user behavior characteristic and the layout of the room. Based on the available location knowledge of a legitimate user (UE) and an eavesdropper (ED), an LED transmitter can choose to convey data or a jamming signal. We call this strategy spatial jamming. By employing a continuous LED model, the related optimization problems are formulated and analyzed based on the signal-tointerference-plus-noise ratio and the secrecy rate, respectively. The numerical results are provided to validate the prediction that the proposed spatial jamming scheme can effectively secure a VLC transmission even when the LEDs do not know the exact location of the ED.
\end{abstract}

Index Terms-Physical layer security, visible light communication, spatial jamming, stochastic geometry, secrecy rate.

\section{INTRODUCTION}

Visible Light Communication (VLC) is an emerging technology that utilizes visible light spectrum from $400 \mathrm{THz}$ to $700 \mathrm{THz}$ as its communication medium. As the demand for data traffic to wireless communication has enormously increased due to the developments of new information technology devices and applications, VLC has gained great popularity in academia and industry as a promising communication technology to resolve the shortfall of radio frequency (RF) spectrum. Compared to the RF systems, VLC has a few notable advantages in terms of unlicensed wide bandwidth, low implementation cost, high area spectral efficiency, and high security, where the last two advantages come from the fact that visible light intrinsically cannot penetrate a wall or other opaque barriers [1].

However, even with the inherent wireless communication security of VLC systems, there is still a possibility that an eavesdropper (ED) can wiretap important/private information in large open spaces, such as libraries, offices, shopping malls, etc. Thus, to secure the transmission in VLC systems, various physical layer security (PLS) techniques have been proposed and studied. PLS constitutes a set of techniques that enable a transmitter and a legitimate receiver (UE) to securely transmit and receive important data by employing channel randomness at the cost of reducing the communication rate [2], [3]. The typical examples of PLS techniques for VLC systems include beamforming, light-emitting diode (LED) selection, friendly

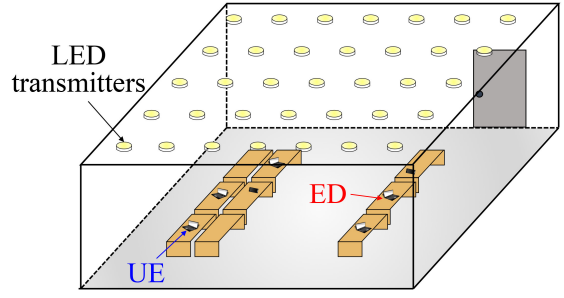

Fig. 1. An indoor VLC network consisting of multiple LED transmitters, one UE and one ED.

jamming, cooperative jamming, etc. [4]-[11]. Especially, the jamming strategies [6]-[11] are very useful for preventing the ED from wiretapping the information since it is not possible to extract only the information component from the received signal if the jamming signal is random.

In addition to the previous works, a practical observation of VLC environments has stimulated us to devise a new jamming strategy. Fig. 1 shows an example of an office installed with a VLC system, where multiple mobile VLC devices are located on fixed desks. Considering the typical behaviors of the office workers in which they mostly work sitting at the desks, it can be assumed that the LED transmitters know the probable and approximate locations of the mobile devices, i.e., near the desks, neither in the aisle nor the rest area ${ }^{1}$. In other words, in practice, the LEDs transmitters are able to know the exact location and/or the CSI of the UE by utilizing a channel estimation method [12], and may have knowledge of the probable and approximate locations of the ED. Besides, as in [8], it also can be assumed that the ED might be a registered user in the network, but, in a certain transmission session, the confidential message needs to be securely delivered from the LEDs to only the intended user; in this case, the LEDs can know the approximate location of the ED. Based on this assumption, in this paper, by utilizing the available information on the locations of the UE and the ED, we propose a spatial jamming strategy in which the LED jammers being located near to the ED emit random jamming signals to hinder the ED's reception of the information signal. Unlike previously proposed jamming schemes [8]-[11], where all the LEDs in the room need to participate in producing a beam-steering

\footnotetext{
${ }^{1}$ Even in other VLC system environments, such as libraries, conference rooms, etc., the possible locations of VLC users also can be anticipated by analyzing the user behavior characteristics and the layout of the room.
} 


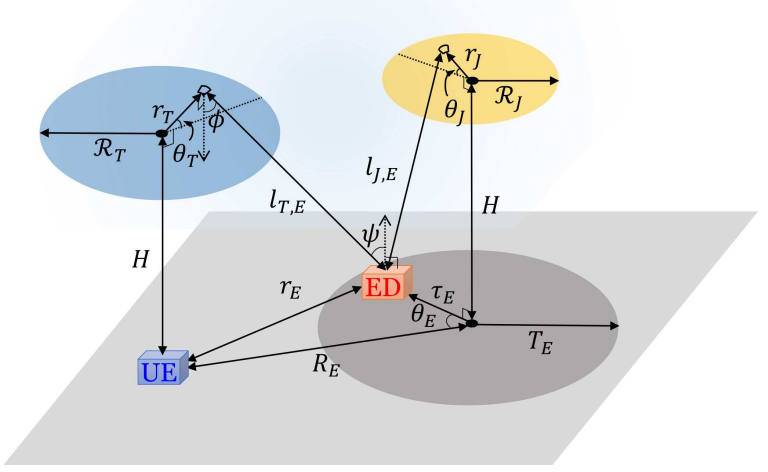

Fig. 2. Geometry of a continuous LED model with the spatial jamming scheme.

strategy, in the spatial jamming strategy, only the adjacent LEDs need to alternatively transmit a data or jamming signal, which would improve the spatial efficiency of VLC systems and permit a more straightforward implementation regarding low synchronization requirement and computational complexity.

The rest of this paper is organized as follows. Section II begins with the system model describing a continuous LED model and providing analysis for the received optical power density. Section III provides the secrecy performance measures. In Section IV, the spatial jamming scheme is proposed by formulating a few related optimization problems. Section $\mathrm{V}$ provides numerical results to validate the proposed scheme. Section VI concludes the paper.

\section{SYSTEM MODEL}

\section{A. Room Configuration with Continuous LEDs}

Rather than considering the discrete LED transmitters as described in Fig. 1, in this paper, we consider a continuous LED model as described in Fig. 2, where an infinite number of LED transmitters are attached to the ceiling of an infinitely large room, and the distances among the LEDs are infinitesimal. As shown in [13], this continuum model significantly simplifies the calculation of the received optical power, while effectively approximating the real LED transmitters, i.e., the discrete model, since LED transmitters in practice are uniformly distributed to illuminate an entire room satisfying the brightness standards of the room from 400 to 1000 [lux] [14].

In this paper, we propose a spatial jamming strategy that utilizes the knowledge of the locations of the UE and the ED. Since the channel gain in VLC systems largely depends on the distance between an LED and a receiver's photodiode (PD) [15], we expect that the LEDs being located near to the UE act as information transmitters, while the LEDs being located near to the ED act as jammers. Therefore, in Fig. 2, assuming that one UE and one ED are present in the work plane (the gray plane), the LEDs transmitting data are denoted by the blue circular disk with radius $\mathcal{R}_{T}$, where the UE is located right below the circle. On the other hand, the LEDs emitting jamming signals are denoted by the yellow circular disk with radius $\mathcal{R}_{J}$, where the ED is located $\tau_{E}$ away from the point right below the yellow circular plane. This assumption can be justified since the information LED transmitters can be accurately selected according to the location of the UE, which is assumed to be known to the LEDs. In contrast, it is assumed that the exact location of the ED is not known to the LEDs, only its approximate location is available; thus, selecting the jammers cannot be accurate. In addition, in this work, we assume that all of the LED transmitters can share the data and jamming signals by wire cables and are capable of selectively transmitting either a data or jamming signal.

\section{B. Received Optical Power Density Analysis}

According to [15], the optical channel gain between an LED transmitter and a receiver in VLC systems can be written as

$$
G=\left\{\begin{array}{cc}
\frac{(m+1)}{2 \pi l^{2}} A_{\mathrm{RX}} \cos ^{m}(\phi) \cos (\psi) & \text { for }|\psi| \leq \Psi, \\
0 & \text { for }|\psi|>\Psi
\end{array}\right.
$$

where $m=-\ln (2) / \ln \left(\cos \left(\phi_{1 / 2}\right)\right)$ is the order of Lambertian emission with half illuminance at $\phi_{1 / 2} . l$ is the distance between the LED and the receiver. $\phi$ and $\psi$ denote the angle of irradiance and the incidence between the transmitter and the receiver, respectively. Also, the receiver collection area is given by $A_{\mathrm{RX}}=\kappa^{2} A_{\mathrm{PD}} / \sin ^{2}(\Psi)$, where $\kappa$ is the refractive index of the optical concentrator, $A_{\mathrm{PD}}$ is the physical area of the PD, and $\Psi$ is the received field of view of the PD. Moreover, as in [4], [5], if we assume that a receiver's PD faces up normal to the work plane, we can rewrite (1) as

$$
G=\frac{(m+1)}{2 \pi l^{2}} A_{\mathrm{RX}}\left(\frac{H}{l}\right)^{m}\left(\frac{H}{l}\right)=\varrho l^{-(m+3)}
$$

where $\varrho=(m+1) A_{\mathrm{RX}} H^{(m+1)} / 2 \pi$. Note that (2) is valid only when $|\psi| \leq \Psi$ is satisfied.

In the continuum model, to deal with the infinite number of LEDs, we characterize the emitted optical power of LEDs by using the optical power density per unit of LED area $P_{T}$ $\left[\mathrm{W} / \mathrm{m}^{2}\right]$. We assume that the information transmitter and the jammer emit signals with the same optical power density $P_{T}$. Utilizing the channel gain model in (2), firstly, the received optical power density of the data signal emitted by the information transmitters (i.e., the blue circular plane) $P_{D}\left[\mathrm{~W} / \mathrm{m}^{2}\right]$ at a point $r_{E}[\mathrm{~m}]$ away from the UE in the work plane can be described by

$$
\begin{gathered}
P_{D}\left(r_{E}\right)=\int_{0}^{\mathcal{R}_{T}} \int_{0}^{2 \pi} P_{T} \frac{(m+1)}{2 \pi l_{T, E}^{2}}\left(\frac{H}{l_{T, E}}\right)^{(m+1)} r_{T} \mathrm{~d} \theta_{T} \mathrm{~d} r_{T} \\
\stackrel{(a)}{=} \frac{P_{T}}{2}\left(1+\frac{\mathcal{R}_{T}^{2}-H^{2}-r_{E}^{2}}{\sqrt{\left(H^{2}+r_{E}^{2}\right)^{2}+2 \mathcal{R}_{T}^{2}\left(H^{2}-r_{E}^{2}\right)+\mathcal{R}_{T}^{4}}}\right)
\end{gathered}
$$

where $l_{T, E}=\sqrt{r_{T}^{2}+r_{E}^{2}-2 r_{T} r_{E} \cos \theta_{T}+H^{2}}$ denotes the distance between the differential information transmitter and the ED. For (a) and the following analysis, we assume all of the LED transmitters have the Lambertian emission pattern with $\phi_{1 / 2}=60^{\circ}(m=1)$. Also, note that $P_{D}(0)$ denotes the received optical power density of the data signal at the UE site, and that $r_{E}$ can be described as a function of $\tau_{E}$ and $\theta_{E}$ as $r_{E}=\sqrt{\tau_{E}^{2}+R_{E}^{2}-2 \tau_{E} R_{E} \cos \theta_{E}}$. 
Secondly, the received optical power density of the jamming signal emitted by the jammers (i.e., the yellow circle) $P_{J}\left[\mathrm{~W} / \mathrm{m}^{2}\right]$ at the ED site, which is $\tau_{E}[\mathrm{~m}]$ away from the right below point of the yellow circular plane in the work plane, can be described by

$$
\begin{aligned}
P_{J}\left(\tau_{E}\right) & =\int_{0}^{\mathcal{R}_{J}} \int_{0}^{2 \pi} P_{T} \frac{(m+1)}{2 \pi l_{J, E}^{2}}\left(\frac{H}{l_{J, E}}\right)^{(m+1)} r_{J} \mathrm{~d} \theta_{J} \mathrm{~d} r_{J} \\
& =\frac{P_{T}}{2}\left(1+\frac{\mathcal{R}_{J}^{2}-H^{2}-\tau_{E}^{2}}{\sqrt{\left(H^{2}+\tau_{E}^{2}\right)^{2}+2 \mathcal{R}_{J}^{2}\left(H^{2}-\tau_{E}^{2}\right)+\mathcal{R}_{J}^{4}}}\right)
\end{aligned}
$$

where $l_{J, E}=\sqrt{r_{J}^{2}+\tau_{E}^{2}-2 r_{J} \tau_{E} \cos \theta_{J}+H^{2}}$ denotes the distance between the differential jammer and the ED. Note that $P_{J}\left(R_{E}\right)$ denotes the received optical power density of the jamming signal at the UE site.

\section{Data and Jamming Transmission}

The data current signal $x(t) \in[-1,1]$ and the jamming signal $j(t) \in[-1,1]$ in time slot $t$ are generated from a certain real constellation, e.g., a DC-biased pulse amplitude modulation (PAM) scheme, and multiplied by a modulation index $\alpha \in[0,1]$ and a fixed bias current $I_{\mathrm{DC}} \in \mathbb{R}^{+}$, where $\mathbb{R}^{+}$ denotes the set of non-negative real-valued numbers. Note that $j(t)$ must be a random value to prevent the ED from canceling the jamming component from the received signal. Thus, the modulated signal $s(t)$ can be described as $s(t)=\alpha I_{\mathrm{DC}} x(t)$ or $s(t)=\alpha I_{\mathrm{DC}} j(t)$. To maintain linear current-to-light conversion and avoid clipping distortion, the LED transmitter has an amplitude constraint on its input power, i.e., $s(t)$ is subject to the amplitude constraint $|s(t)| \leq \alpha I_{\mathrm{DC}}$. Therefore, the emitted optical power of each LED can be $P_{\mathrm{TX}}(t)=\eta\left(I_{\mathrm{DC}}+s(t)\right)$, where $\eta[W / A]$ is the current-to-light conversion efficiency. The optical power density $P_{T}$ for the continuum model can be related to the emitted optical power of each LED $P_{\mathrm{TX}}(t)$ with $P_{T}=\lambda_{T} \mathbb{E}\left[P_{\mathrm{TX}}(t)\right]=\lambda_{T} \eta I_{\mathrm{DC}}$, where $\lambda_{T}$ is the density of LED transmitters. Also, $\mathbb{E}[s(t)]=0$ is assumed, the modulated signal does not affect illumination.

Utilizing the optical power density expressions (3) and (4), the received signal voltage $y_{k}(t)$ after removing the DC bias, where $k \in\{U, E\}$ denotes the index number of the UE and $\mathrm{ED}$, respectively, can be described as

$$
\begin{gathered}
y_{U}(t)=\zeta_{U} P_{D}(0) \hat{x}(t)+\zeta_{U} P_{J}\left(R_{E}\right) \hat{j}(t)+n_{U}(t) \\
y_{E}(t)=\zeta_{E} P_{D}\left(r_{E}\right) \hat{x}(t)+\zeta_{E} P_{J}\left(\tau_{E}\right) \hat{j}(t)+n_{E}(t)
\end{gathered}
$$

respectively, where $\zeta_{k}=\alpha A_{\mathrm{PD}, k} \kappa_{k}^{2} R_{\mathrm{rsp}, k} T_{k} / \sin ^{2}\left(\Psi_{k}\right) \quad\left[\mathrm{m}^{2}\right.$. $\mathrm{V} / \mathrm{W}]$ and $\hat{x}(t)=x(t) /(1[\mathrm{~A}])$ and $\hat{j}=j(t) /(1[\mathrm{~A}])$ are the dimensionless versions of $x(t)$ and $j(t) . R_{\mathrm{rsp}, k} \quad[\mathrm{~A} / \mathrm{W}]$ is the photodetector's responsivity and $T_{k}$ [V/A] is the transimpedance amplifier gain. Also, $n_{k}(t)$ signifies zero-mean additive white Gaussian noise (AWGN) with variance $\sigma^{2}$. For notational convenience, the time index $t$ is ignored for the remainder of the paper.

\section{Performance Measures}

For Gaussian VLC channels with amplitude constraints, we define the peak signal-to-interference-plus-noise ratio (SINR), rather than the average, by assuming $x=1$ and $j=1$ since the channel capacity bounds of the VLC systems are described as a function of the peak SINR [4], [16]. The peak SINRs at the $\mathrm{UE}$ and the ED can be written as

$$
\gamma_{U}=\frac{\zeta_{U}^{2} P_{D}^{2}(0)}{\zeta_{U}^{2} P_{J}^{2}\left(R_{E}\right)+\sigma^{2}}, \quad \gamma_{E}=\frac{\zeta_{E}^{2} P_{D}^{2}\left(r_{E}\right)}{\zeta_{E}^{2} P_{J}^{2}\left(\tau_{E}\right)+\sigma^{2}}
$$

respectively. We use the SINR to denote the peak SINR for the remainder of the paper.

The secrecy capacity of the VLC channel is given by [2]

$$
C_{s}=\max _{p_{X}}\left(\mathbb{I}\left(x ; y_{U}\right)-\mathbb{I}\left(x ; y_{E}\right)\right), \quad \text { s.t. }|x| \leq 1
$$

where $p_{x}$ is the input distribution and $\mathbb{I}(\cdot ; \cdot)$ denotes the mutual information. In VLC systems, since it is infeasible to derive a closed-form expression for the secrecy capacity due to the amplitude constraint [17], we provide an achievable secrecy rate expression for the proposed spatial jamming scheme. To simplify deriving a closed-form achievable secrecy rate expression, we assume that both the data signal $x$ (and $\hat{x}$ ) and the jamming signal $j$ (and $\hat{j}$ ) follow the truncated Gaussian distribution $\mathcal{N}_{T}\left(0, \sigma_{T}^{2}\right)$ defined over $[-1,1]$, where $\sigma_{T} \in \mathbb{R}_{+}$, as in [10]. Its probability density function (PDF) is given by

$$
f(x)=\frac{\phi\left(\frac{x}{\sigma_{T}}\right)}{\Phi\left(\frac{1}{\sigma_{T}}\right)-\Phi\left(\frac{-1}{\sigma_{T}}\right)}
$$

where $\phi(v)=e^{-v^{2} / 2} / \sqrt{2 \pi}$ and $\Phi(\omega)=(1+\operatorname{erf}(\omega / \sqrt{2})) / 2$. The error function $\operatorname{erf}(\cdot)$ is defined as $\operatorname{erf}(\omega)=1 / \sqrt{\pi} \int_{-\omega}^{\omega} e^{-t^{2}} \mathrm{~d} t$. Note that the optimal input distribution under the amplitude constraint in VLC systems is not readily available, and the truncated Gaussian distribution was shown to outperform the uniform distribution in the terms of secrecy rates in VLC systems [10]. With this assumption, we present the following lemma, which provides an analytic achievable secrecy rate expression for the system in question.

Lemma 1. An achievable secrecy rate for the Gaussian wiretap channel in (5) with the spatial jamming scheme can be obtained by lower-bounding the secrecy capacity in (7) to give

$$
\begin{aligned}
R_{s}= & \max \left\{\frac{1}{2} \log \left(\frac{e^{2 \eta}\left(P_{D}^{2}(0)+P_{J}^{2}\left(R_{E}\right)\right)+C}{\varphi P_{J}^{2}\left(R_{E}\right)+C}\right)\right. \\
& \left.-\frac{1}{2} \log \left(\frac{\varphi\left(P_{D}^{2}\left(r_{E}\right)+P_{J}^{2}\left(\tau_{E}\right)\right)}{e^{2 \eta} P_{J}^{2}\left(\tau_{E}\right)}\right), 0\right\}
\end{aligned}
$$

where

$$
\begin{aligned}
& \eta=\log (Z)+\frac{-\frac{1}{\sigma_{T}} \phi\left(\frac{-1}{\sigma_{T}}\right)-\frac{1}{\sigma_{T}} \phi\left(\frac{1}{\sigma_{T}}\right)}{2 Z} \\
& \varphi=1+\frac{\frac{-1}{\sigma_{T}} \phi\left(\frac{-1}{\sigma_{T}}\right)-\frac{1}{\sigma_{T}} \phi\left(\frac{1}{\sigma_{T}}\right)}{Z}-\left(\frac{\phi\left(\frac{-1}{\sigma_{T}}\right)-\phi\left(\frac{1}{\sigma_{T}}\right)}{Z}\right)^{2}, \\
& Z=\Phi\left(\frac{1}{\sigma_{T}}\right)-\Phi\left(\frac{-1}{\sigma_{T}}\right), \quad C=\sigma^{2} / \zeta_{U}^{2} \sigma_{T}^{2}
\end{aligned}
$$

Proof. See Appendix A. 


\section{SPATIAL JAMMING}

In this section, we investigate optimization problems with the spatial jamming strategy based on $\mathrm{SINR}^{2}$ and the secrecy rate objectives. In the first subsection, we analyze the optimization problem assuming the LEDs know the exact locations of both the UE and the $\mathrm{ED}^{3}$. On the other hand, in the second subsection, we consider a scenario that the exact location of the UE and the approximate location of the ED, which is obtained from investigating the layout of the room and the typical behavior of the workers, are known to the LEDs.

\section{A. One Legitimate User and One Known Eavesdropper}

In this subsection, we assume that the LEDs know the exact locations of the UE and the ED. In other words, it is assumed that $\tau_{E}$ and $\theta_{E}$ are given to the LEDs.

1) Optimization Based on SINR: A natural objective of the optimization problem based on the SINR can be to maximize the SINR of the UE $\gamma_{U}$ subject to a constraint on the SINR of the ED $\gamma_{E}$. Given the exact locations of the UE and the $\mathrm{ED}$, the optimization problem of $\mathcal{R}_{T}$ and $\mathcal{R}_{J}$ for the spatial jamming can be formulated as

$$
\gamma_{U}^{\star}=\max _{\mathcal{R}_{T}, \mathcal{R}_{J}} \frac{\zeta_{U}^{2} P_{D}^{2}(0)}{\zeta_{U}^{2} P_{J}^{2}\left(R_{E}\right)+\sigma^{2}}, \quad \text { s.t. }\left\{\begin{array}{l}
\frac{\zeta_{E}^{2} P_{D}^{2}\left(r_{E}\right)}{\zeta_{E}^{2} P_{J}^{2}\left(\tau_{E}\right)+\sigma^{2}}<\rho_{E} \\
\mathcal{R}_{T}+\mathcal{R}_{J} \leq R_{E}
\end{array}\right.
$$

where $\rho_{E}$ is the target constraint on $\gamma_{E}$. The second constraint ensures that the two information and jamming LED circles do not overlap. This optimization problem is a non-convex problem. However, the fact that the problem consists of two optimization variables $\mathcal{R}_{T}$ and $\mathcal{R}_{J}$ alleviates the difficulty in finding the optimal solution. In practice, finding the optimal solution of the two parameters can be executed by using Sequential Quadratic Programming (SQP) [18] in a second on a standard PC (Intel i7, $3.4 \mathrm{GHz}$ ) using MATLAB.

2) Optimization Based on Secrecy Rate: From (9), we formulate the optimization problem of $\mathcal{R}_{T}$ and $\mathcal{R}_{J}$ maximizing the secrecy rate under the spatial jamming strategy as

$$
R_{s}^{\star}=\max _{\mathcal{R}_{T}, \mathcal{R}_{J}} R_{S}, \quad \text { s.t. } \mathcal{R}_{T}+\mathcal{R}_{J} \leq R_{E} .
$$

This optimization problem is also non-convex, however, finding the solution of the optimization problem with only two optimization variables is straightforward, similar to (10).

\section{B. One Legitimate User and One Random Eavesdropper}

In this subsection, we assume that the LEDs know the exact location of the UE and the approximate and probable location of the ED, i.e., the joint PDF of $\left(\tau_{E}, \theta_{E}\right)$ is given to the LEDs.

1) Optimization Based on SINR: Without knowledge of the exact location of the ED, a natural objective is to maximize the SINR of the UE $\gamma_{U}$, subject to a constraint on the average SINR of the ED $\mathbb{E}\left[\gamma_{E}\right]$. Assuming that an ED is randomly

\footnotetext{
${ }^{2}$ Although the SINR expressions in (6) do not directly link to the secrecy rate expression in (9), solely considering the SINRs of the UE and ED is also useful from secrecy perspective as in [5]-[7].

${ }^{3}$ This assumption may be limited in practice, but it is still worthy to be investigated to see how the distance between the center of the jamming circle and the ED, i.e., $\tau_{E}$, would affect the performance of the spatial jamming.
}

located in a circle with radius $T_{E}$, i.e., the dark gray circle in Fig. 2, whose center point is same to that of the yellow circle, the average SINR of an ED can be written as

$$
\bar{\gamma}_{E}=\mathbb{E}\left[\gamma_{E}\right]=\int_{0}^{T_{E}} \int_{0}^{2 \pi} f_{\tau_{E}, \theta_{E}}(\tau, \theta) \frac{\zeta_{E}^{2} P_{D}^{2}\left(r_{E}\right)}{\zeta_{E}^{2} P_{J}^{2}(\tau)+\sigma^{2}} \tau \mathrm{d} \theta \mathrm{d} \tau
$$

where $r_{E}=\sqrt{\tau^{2}+R_{E}^{2}-2 \tau R_{E} \cos \theta}$ and $f_{\tau_{E}, \theta_{E}}(\tau, \theta)$ is the joint PDF of $\left(\tau_{E}, \theta_{E}\right)$. For example, $f_{\tau_{E}, \theta_{E}}(\tau, \theta)$ for the uniform distribution and the bivariate normal distribution of the ED location can be written as

$$
\begin{aligned}
& f_{\tau_{E}, \theta_{E}}^{\text {unif. }}(\tau, \theta)=\frac{1}{\pi T_{E}^{2}} \text { for }\left\{\begin{array}{l}
0 \leq \tau<T_{E} \\
0 \leq \theta<2 \pi
\end{array}\right. \\
& f_{\tau_{E}, \theta_{E}}^{\text {norm. }}(\tau, \theta)=\frac{1}{2 \pi \sigma_{E}^{2}} \exp \left(\frac{-\tau^{2}}{2 \sigma_{E}^{2}}\right) \text { for }\left\{\begin{array}{l}
0 \leq \tau<\infty \\
0 \leq \theta<2 \pi
\end{array}\right.
\end{aligned}
$$

respectively, where $\sigma_{E}^{2}$ in (13b) is the variance of the ED location in a Cartesian coordinate system, i.e., $x=\tau \cos \theta$ and $y=\tau \sin \theta$ with $X \sim \mathcal{N}\left(0, \sigma_{E}\right), Y \sim \mathcal{N}\left(0, \sigma_{E}\right)$ and their correlation is assumed to be as $\operatorname{cor}(X, Y)=0$.

Utilizing this average SINR of the ED, we formulate the optimization problem as

$$
\gamma_{U}^{*}=\max _{\mathcal{R}_{T}, \mathcal{R}_{J}} \frac{\zeta_{U}^{2} P_{D}^{2}(0)}{\zeta_{U}^{2} P_{J}^{2}\left(R_{E}\right)+\sigma^{2}}, \quad \text { s.t. }\left\{\begin{array}{l}
\bar{\gamma}_{E}<\bar{\rho}_{E} \\
\mathcal{R}_{T}+\mathcal{R}_{J} \leq R_{E}
\end{array}\right.
$$

where $\bar{\rho}_{E}$ is the target constraint on $\bar{\gamma}_{E}$. Note that the optimization problem (14) is also a non-convex problem, and $\bar{\gamma}_{E}$ in the first constraint includes the integration to be solved numerically. However, the facts that the number of the optimization variables is only two and $\bar{\gamma}_{E}$ includes only a two-dimensional integral enable the problem to be solved numerically in an efficient manner. In practice, finding the optimal solutions of the two parameters can be executed by using $\mathrm{SQP}$ in a few seconds on a standard PC using MATLAB.

2) Optimization Based on Secrecy Rate: Similarly, the average secrecy rate can be calculated by numerically evaluating

$$
\begin{aligned}
\bar{R}_{S}= & \mathbb{E}\left[R_{S}\right]=\int_{0}^{T_{E}} \int_{0}^{2 \pi} f_{\tau_{E}, \theta_{E}}(\tau, \theta) R_{S}(\tau, \theta) \tau \mathrm{d} \theta \mathrm{d} \tau \\
= & \int_{0}^{T_{E}} \int_{0}^{2 \pi} f_{\tau_{E}, \theta_{E}}(\tau, \theta) \max \left\{\frac{1}{2} \log \left(\frac{e^{2 \eta}\left(P_{D}^{2}(0)+P_{J}^{2}\left(R_{E}\right)\right)+C}{\varphi P_{J}^{2}\left(R_{E}\right)+C}\right)\right. \\
& \left.-\frac{1}{2} \log \left(\frac{\varphi\left(P_{D}^{2}\left(r_{E}\right)+P_{J}^{2}(\tau)\right)}{e^{2 \eta} P_{J}^{2}(\tau)}\right), 0\right\} \tau \mathrm{d} \theta \mathrm{d} \tau .
\end{aligned}
$$

Utilizing (15), the optimization problem maximizing the $a v$ erage secrecy rate can be formulated as

$$
\bar{R}_{s}^{*}=\max _{\mathcal{R}_{T}, \mathcal{R}_{J}} \bar{R}_{s}, \quad \text { s.t. } \mathcal{R}_{T}+\mathcal{R}_{J} \leq R_{E} .
$$

This optimization problem is also non-convex, however, due to the similar reasons related to (14), finding the optimal solution is straightforward such that it can be executed in a few seconds. 


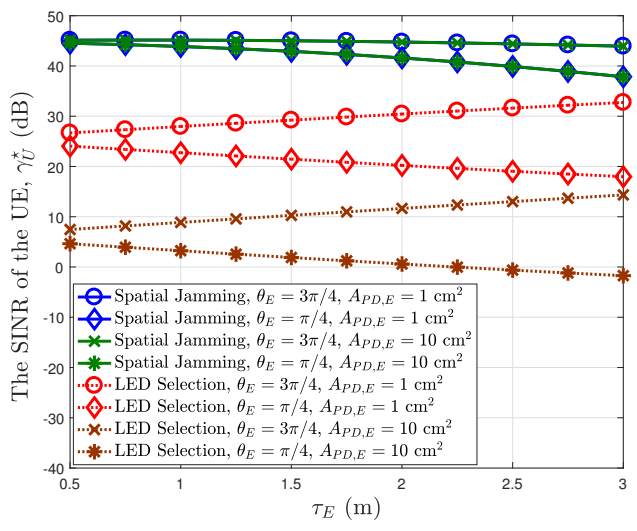

(a) The SINR of the UE $\gamma_{U}^{\star}$ (10).

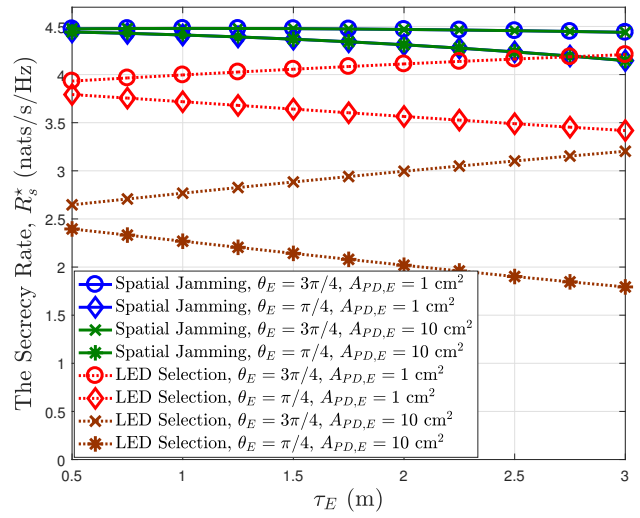

(b) The secrecy rate $R_{S}^{\star}(11)$.

Fig. 3. The optimized SINR of the UE and the secrecy rate for different locations of the ED, i.e., the knowledge of $\left(\tau_{E}, \theta_{E}\right)$ is given to the LED transmitters. The result for the LED selection scheme [5] is given as a benchmark. $\rho_{E}=0.01$ is used.

\section{NumERicAl RESUlts}

In this section, numerical results are given to verify the performance of the proposed spatial jamming scheme. We set $H=2.2 \mathrm{~m}, P_{T}=1 \mathrm{~W} / \mathrm{m}^{2}, \alpha=0.5, \kappa=1.5, A_{\mathrm{PD}, U}=1 \mathrm{~cm}^{2}$, $R_{E}=8 \mathrm{~m}, \Psi=90^{\circ}, \sigma^{2}=1.47 \times 10^{-13} A^{2}$, and $\sigma_{T}=0.62$.

Figs. 3 (a) and (b) show the optimized SINR of the UE and the optimized secrecy rate obtained from solving (10) and (11), respectively, when the LEDs retain the knowledge of the exact locations of both the UE and the ED. The result for the LED selection (without jamming) [5] is given as a benchmark by transforming it into the continuum LED model. The optimal radius of LEDs is found out in similar ways. When the ED moves away from the UE as well as the center of the jamming circle, i.e., when $\tau_{E}$ with $\theta_{E}=3 \pi / 4$ increases, the SINR of the UE and the secrecy rate remain almost unchanged. This result comes from the fact that although the jamming signal at the ED site decreases as $\tau_{E}$ increases, the amount of the received data signal of the ED also decreases as $r_{E}$ increases. In contrast, when the ED moves closer to the UE while moving away from the center of the jamming circle, i.e., when $\tau_{E}$ with $\theta_{E}=\pi / 4$ increases, it is noted that both the SINR of the UE and the secrecy rate slightly decrease. Also, compared to the benchmark, it is shown that the spatial jamming scheme outperforms the LED selection

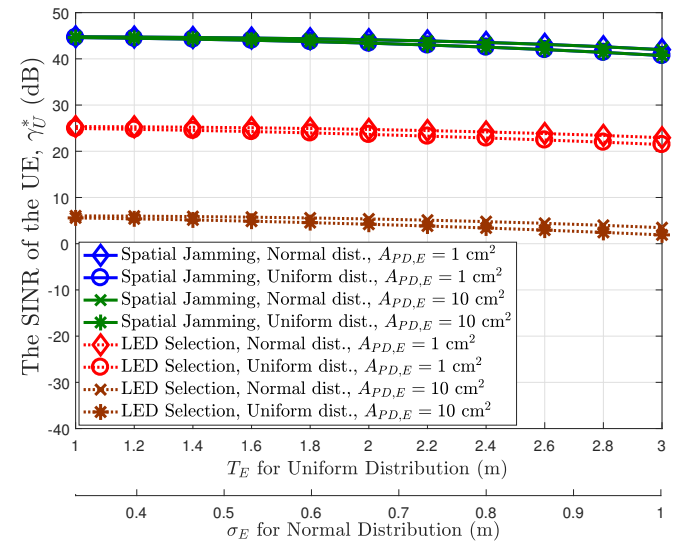

(a) The SINR of the UE $\gamma_{U}^{*}$ (14).

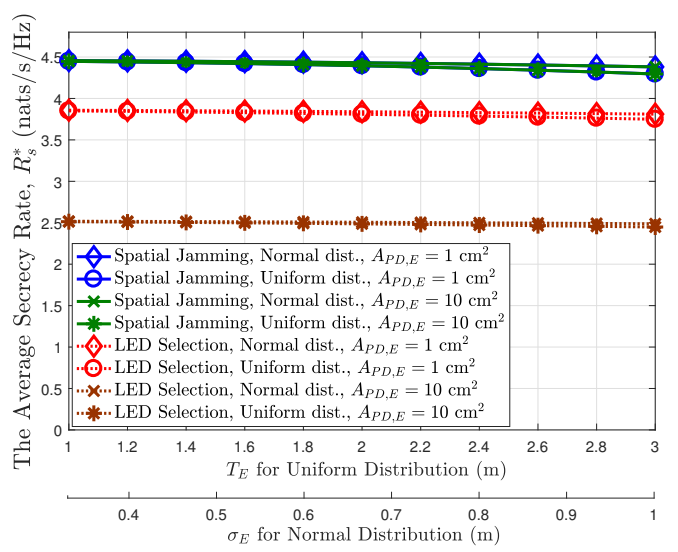

(b) The average secrecy rate $\bar{R}_{S}^{*}(16)$.

Fig. 4. The optimized SINR of the UE and the secrecy rate for different distribution of the ED. The uniform distribution in (13a) and the bivariate normal distribution in (13b) are used. The result for the LED selection scheme [5] is given as a benchmark. $\bar{\rho}_{E}=0.01$ is used.

over the whole region of $\tau_{E}$ for both values of $\theta_{E}$. Moreover, the performance gap between these two schemes becomes more significant when the physical area of the PD for the $\mathrm{ED}\left(A_{\mathrm{PD}, E}=10 \mathrm{~cm}^{2}\right)$ is set much larger than that of the UE $\left(A_{\mathrm{PD}, U}=1 \mathrm{~cm}^{2}\right)^{4}$. This is because the ED can receive more data signal by using a larger PD under the LED selection, however, under the spatial jamming scheme, the ED receives more of the jamming signals as well as the information signals through the larger PD.

Figs. 4 (a) and (b) show the optimized SINR of the UE and the optimized average secrecy rate obtained from solving (14) and (16), respectively, when the exact UE location and the statistical information about the ED location are available. The two joint PDFs of $\left(\tau_{E}, \theta_{E}\right)$ for the uniform and bivariate distributions in (13) are used. Similarly, the LED selection is given as a benchmark. In Figs. 4 (a) and (b), it is shown that the SINR of the UE and the average secrecy rate with the spatial jamming scheme very slightly decrease as $T_{E}$ and $\sigma_{E}$ increase (i.e., the ED is more likely to be located far

\footnotetext{
${ }^{4}$ Note that increasing the physical area of the ED's PD is one of the feasible and simple ways to improve its reception capability, which allows the ED to be located far away from the UE escaping the vigilance of the UE.
} 
away from the center of the jamming circle). Also, the fact that the gaps between the normal and uniform distributions are not significant for all settings indicates that the proposed jamming scheme doesn't require the exact distribution of the ED's location. Moreover, the performance gap between the proposed spatial jamming and the LED selection is very large, even when the LEDs do not hold the knowledge of the exact location of the ED. This result validates the prediction that even when the LEDs do not know the exact location of the ED, the proposed spatial jamming scheme effectively suppresses the information reception of the ED, which improves the SINR of the UE and the average secrecy rate.

\section{CONCLUSION}

In this paper, a novel spatial jamming strategy utilizing the available knowledge of the UE and the ED was proposed. Related optimization problems based on the SINR and the secrecy rate were formulated. By employing the continuous LED model, the optimization problems could be significantly simplified, such that they could be solved numerically in an efficient manner. Numerical simulations verified that, even when the LEDs only know the approximate location of the ED, the proposed spatial jamming scheme can effectively secure the transmission in VLC systems.

\section{APPENDIX A}

\section{Derivation of the Secrecy Rate with Spatial JAMMING}

A lower bound of (7) can be obtained as

$$
\begin{aligned}
C_{S} & =\max _{p_{x}, p_{j}}\left(\mathbb{I}\left(x ; y_{U}\right)-\mathbb{I}\left(x ; y_{E}\right)\right)=\max _{p_{\hat{x}}, p_{\hat{j}}}\left(\mathbb{I}\left(\hat{x} ; y_{U}\right)-\mathbb{I}\left(\hat{x} ; y_{E}\right)\right) \\
& \stackrel{(a)}{\geq} \mathbb{I}\left(\hat{x} ; y_{U}\right)-\mathbb{I}\left(\hat{x} ; y_{E}\right) \stackrel{(b)}{\geq} \mathbb{I}\left(\hat{x} ; y_{U}\right)-\mathbb{I}\left(\hat{x} ; v_{E}\right) \\
& =\mathbb{h}\left(y_{U}\right)-\mathbb{h}\left(y_{U} \mid \hat{x}\right)-\mathbb{h}\left(v_{E}\right)+\mathbb{h}\left(v_{E} \mid \hat{x}\right)
\end{aligned}
$$

where $\mathrm{h}(\cdot)$ denotes differential entropy and $v_{E}=\zeta_{E} P_{D}\left(r_{E}\right) \hat{x}+$ $\zeta_{E} P_{J}\left(\tau_{E}\right) \hat{j}$. (a) follows from dropping the maximization by choosing a truncated Gaussian distribution on $p_{\hat{x}}$ and $p_{\hat{j}}$, and (b) follows from the data-processing inequality, i.e., $y_{E}=$ $g\left(v_{E}\right)=v_{E}+n_{E}$. Firstly, we lower-bound $\operatorname{h}\left(y_{U}\right)$ by using the entropy-power inequality as

$$
\begin{aligned}
& \mathbb{h}\left(y_{U}\right) \geq \frac{1}{2} \log \left(e^{2 \mathfrak{h}\left(\zeta_{U} P_{D}(0) \hat{x}\right)}+e^{2 \mathfrak{h}\left(\zeta_{U} P_{J}\left(R_{E}\right) \hat{j}\right)}+e^{2 \mathrm{~h}\left(n_{U}\right)}\right) \\
& \quad=\frac{1}{2} \log \left(2 \pi e\left(\sigma_{T}^{2} e^{2 \eta} \zeta_{U}^{2}\left(P_{D}^{2}(0)+P_{J}^{2}\left(R_{E}\right)\right)+\sigma^{2}\right)\right)
\end{aligned}
$$

where (18) follows from the facts that

$$
\begin{aligned}
& \operatorname{lh}\left(\zeta_{U} P_{D}(0) \hat{x}\right)=\log \left(\zeta_{U} P_{D}(0)\right)+\frac{1}{2} \log \left(2 \pi e \sigma_{T}^{2}\right)+\eta, \\
& \mathbb{h}\left(\zeta_{U} P_{J}\left(R_{E}\right) \hat{j}\right)=\log \left(\zeta_{U} P_{J}\left(R_{E}\right)\right)+\frac{1}{2} \log \left(2 \pi e \sigma_{T}^{2}\right)+\eta, \\
& \mathbb{h}\left(n_{U}\right)=\frac{1}{2} \log 2 \pi e \sigma^{2} .
\end{aligned}
$$

Then, we upper-bound $\mathrm{h}\left(y_{U} \mid x\right)$ and $\mathrm{h}\left(v_{E}\right)$ as

$$
\begin{aligned}
\mathbb{h}\left(y_{U} \mid \hat{x}\right)= & \mathbb{h}\left(y_{U}-\zeta_{U} P_{D}(0) \hat{x} \mid \hat{x}\right)=\mathbb{h}\left(\zeta_{U} P_{J}\left(R_{E}\right) \hat{j}+n_{U}\right) \\
\leq & \frac{1}{2} \log 2 \pi e\left(\sigma_{T}^{2} \zeta_{U}^{2} \varphi P_{J}^{2}\left(R_{E}\right)+\sigma^{2}\right) \\
\mathbb{h}\left(v_{E}\right) & =\mathbb{h}\left(\zeta_{E} P_{D}\left(r_{E}\right) \hat{x}+\zeta_{E} P_{J}\left(\tau_{E}\right) \hat{j}\right) \\
& \leq \frac{1}{2} \log 2 \pi e\left(\sigma_{T}^{2} \zeta_{E}^{2} \varphi\left(P_{D}^{2}\left(r_{E}\right)+P_{J}^{2}\left(\tau_{E}\right)\right)\right)
\end{aligned}
$$

by using the differential entropy of Gaussian random variables with variances $\mathbb{v a r}\left\{\zeta_{U} P_{J}\left(R_{E}\right) J+N_{U}\right\}$ and $\mathbb{v a r}\left\{\zeta_{E} P_{D}\left(r_{E}\right) X+\right.$ $\left.\zeta_{E} P_{J}\left(\tau_{E}\right) J\right\}$, respectively. Lastly, we have

$$
\begin{aligned}
\mathbb{h}\left(v_{E} \mid \hat{x}\right) & =\mathbb{h}\left(v_{E}-\zeta_{E} P_{D}\left(r_{E}\right) \hat{x} \mid \hat{x}\right)=\mathbb{h}\left(\zeta_{E} P_{J}\left(\tau_{E}\right) \hat{j}\right) \\
& =\frac{1}{2} \log \left(2 \pi e \sigma_{T}^{2} \zeta_{E}^{2} P_{J}^{2}\left(\tau_{E}\right)\right)+\eta
\end{aligned}
$$

Plugging (18), (20), (21) and (22) into (17) yields the secrecy rate for the spatial jamming technique in (9).

\section{ACKNOWLEDGMENT}

The work was supported in part by EU Horizon 2020 grant number 761992 ("IoRL"), EPSRC grant number EP/N002350/1 ("Spatially Embedded Networks") and EP/R006377/1 ("M3NETs").

\section{REFERENCES}

[1] H. Haas, L. Yin, Y. Wang, and C. Chen, "What is LiFi?" J. Lightw. Technol., vol. 34, no. 6, pp. 1533-1544, Mar. 2016.

[2] M. Bloch and J. Barros, Physical-Layer Security: From Information Theory to Security Engineering. Cambridge University Press, 2011.

[3] G. Chen, J. P. Coon, and M. D. Renzo, "Secrecy outage analysis for downlink transmissions in the presence of randomly located eavesdroppers," IEEE Trans. Inf. Forens. Security, vol. 12, no. 5, pp. 1195-1206, May 2017.

[4] A. Mostafa and L. Lampe, "Physical-layer security for MISO visible light communication channels," IEEE J. Sel. Areas Commun., vol. 33 , no. 9, pp. 1806-1818, Sep. 2015

[5] S. Cho, G. Chen, and J. P. Coon, "Securing visible light communication systems by beamforming in the presence of randomly distributed eavesdroppers," IEEE Trans. Wireless Commun., vol. 17, no. 5, pp. 29182931, May 2018.

[6] T. V. Pham, T. Hayashi, and A. T. Pham, "Artificial-noise-aided precoding design for multi-user visible light communication channels," IEEE Access, vol. 7, pp. 3767-3777, 2019.

[7] H. Shen, Y. Deng, W. Xu, and C. Zhao, "Secrecy-oriented transmitter optimization for visible light communication systems," IEEE Photon. J, vol. 8, no. 5, 2016.

[8] A. Mostafa and L. Lampe, "Securing visible light communications via friendly jamming," in IEEE Globecom Workshops in Austin, USA, Dec. 2014, pp. 524-529.

[9] _ , "Physical-layer security for indoor visible light communications," in IEEE ICC in Sydney, Australia, Jun. 2014, pp. 3342-3347.

[10] H. Zaid, Z. Rezki, A. Chaaban, and M. S. Alouini, "Improved achievable secrecy rate of visible light communication with cooperative jamming," in IEEE GlobalSIP in Orlando, U.S.A., Dec. 2015, pp. 1165-1169.

[11] F. Wang, C. Liu, Q. Wang, J. Zhang, R. Zhang, L. Yang, and L. Hanzo, "Optical jamming enhances the secrecy performance of the generalized space-shift-keying-aided visible-light downlink," IEEE Trans. Commun., vol. 66, no. 9, pp. 4087-4102, Sep. 2018.

[12] M. Biagi, T. Borogovac, and T. D. C. Little, "Adaptive receiver for indoor visible light communications," J. Lightw. Technol., vol. 31, no. 23, pp. 3676-3686, Dec. 2013.

[13] S. Cho, G. Chen, H. Chun, J. P. Coon, and D. O'Brien, "Impact of multipath reflections on secrecy in VLC systems with randomly located eavesdroppers," in IEEE WCNC in Barcelona, Spain, Apr. 2018, pp. $1-6$.

[14] Lighting of Indoor Work Places, European Stand. EN 12464-1, 2003.

[15] T. Komine and M. Nakagawa, "Fundamental analysis for visible-light communication system using LED lights," IEEE Trans. Consum. Electron., vol. 50, no. 1, pp. 100-107, Feb. 2004.

[16] A. Lapidoth, S. Moser, and M. Wigger, "On the capacity of free-space optical intensity channels," IEEE Trans. Inf. Theory, vol. 55, no. 10, pp. 4449-4461, Oct. 2009.

[17] O. Ozel, E. Ekrem, and S. Ulukus, "Gaussian wiretap channel with an amplitude constraint," in IEEE Inf. Theory Workshop in Lausanne, Switzerland, Sep. 2012, pp. 5553-5563.

[18] P. E. Gill and E. Wong, "Sequential quadratic programming methods," in Mixed Integer Nonlinear Programming, J. Lee and S. Leyffer, Eds. New York, NY: Springer New York, 2012, pp. 147-224. 